\title{
On multipoint resonant problems on the half-line
}

\author{
Lucía López-Somoza ${ }^{1 *}$ (1) and Feliz Minhós²
}

*Correspondence:
lucia.lopez.somoza@usc.es
'Departamento de Estatística,
Análise Matemática e Optimización
Instituto de Matemáticas, Facultade
de Matemáticas, Universidade de
Santiago de Compostela, Galicia,
Spain
Full list of author information is
available at the end of the article

available at the end of the article

\begin{abstract}
In this work we obtain sufficient conditions for the existence of bounded solutions of a resonant multipoint second-order boundary value problem, with a fully differential equation.

The noninvertibility of the linear part is overcome by a new perturbation technique, which allows obtaining an existence result and a localization theorem. Our hypotheses are clearly much less restrictive than those existent in the literature and, moreover, they can be applied to higher-order, resonant or nonresonant, boundary value problems defined on the half-line or even on the real line.
\end{abstract}

Keywords: Multipoint problems; Unbounded domains; Resonant problems

\section{Introduction}

In this paper, we will prove the existence of bounded solutions for the multipoint boundary value problem

$$
\left\{\begin{array}{l}
u^{\prime \prime}(t)=f\left(t, u(t), u^{\prime}(t)\right), \quad t \in[0, \infty) \\
u(0)=0, \quad u^{\prime}(\infty)=\sum_{i=1}^{m-1} \alpha_{i} u^{\prime}\left(\xi_{i}\right)
\end{array}\right.
$$

where $\alpha_{i}>0$ and $0=\xi_{1}<\cdots<\xi_{m-1}<\infty$. We assume that coefficients $\alpha_{i}$ satisfy the resonance condition

$$
\sum_{i=1}^{m-1} \alpha_{i}=1
$$

A boundary value problem is said to be resonant when the correspondent homogeneous problem has nontrivial solutions. In fact, under condition (2), the homogeneous boundary value problem related to (1), namely

$$
\left\{\begin{array}{l}
u^{\prime \prime}(t)=0, \quad t \in[0, \infty) \\
u(0)=0, \quad u^{\prime}(\infty)=\sum_{i=1}^{m-1} \alpha_{i} u^{\prime}\left(\xi_{i}\right)
\end{array}\right.
$$

has a nontrivial solution.

These resonant problems have been studied for many years by many methods: degree theory has been used in, for instance, [3, 7, 13, 22], Lyapunov-Schmidt arguments in [17],

(c) The Author(s) 2019. This article is distributed under the terms of the Creative Commons Attribution 4.0 International License (http://creativecommons.org/licenses/by/4.0/), which permits unrestricted use, distribution, and reproduction in any medium, provided you give appropriate credit to the original author(s) and the source, provide a link to the Creative Commons license, and indicate if changes were made. 
a Leggett-Williams theorem in $[8,21]$, fixed point and fixed point index theories in $[2,9$, $10,25]$, monotone method together with the upper and lower solutions technique in [20], among others.

Boundary value problems on unbounded intervals arise in many models of applied mathematics, such as in combustion theory, in plasma physics, to model the unsteady flow of a gas through semi-infinite porous media, to study the electrical potential of an isolated neutral atom, etc. For more details, techniques and applications in this field we refer, for example, to [11, 14-16, 24], and the monograph [1].

From a theoretical point of view, resonance problems can be formulated by an equation $L x=N x$, where $L$ is a noninvertible operator. Therefore, in particular, the resonance condition (2) implies that the Green's function related to problem (3) does not exist. This issue is overcome applying several techniques. For instance, in [12] the authors studied the problem

$$
\left\{\begin{array}{l}
u^{\prime \prime}(t)+f(t, u(t))=0, \quad t \in[0, \infty), \\
u(0)=0, \quad u^{\prime}(\infty)=\sum_{i=1}^{m-1} \alpha_{i} u^{\prime}\left(\xi_{i}\right),
\end{array}\right.
$$

also under condition (2) and, to deal with the resonance problem, they defined some suitable operators and were able to find a solution in the space

$$
E=\left\{u \in \mathcal{C}[0, \infty), u(0)=0, \sup _{t \in[0, \infty)} \frac{|u(t)|}{1+t}<\infty\right\}
$$

so, clearly, that solution could be unbounded.

Our arguments apply a different technique to find bounded solutions for problem (1). Moreover, we note that, on the contrary to [12], we allow the nonlinearity $f$ to depend on the first derivative of $u$.

In [6], a similar third order boundary value problem is considered, namely

$$
\left\{\begin{array}{l}
u^{\prime \prime \prime}(t)=f\left(t, u(t), u^{\prime}(t), u^{\prime \prime}(t)\right)=0, \quad t \in[0, \infty), \\
u(0)=u^{\prime}(0)=0, \quad u^{\prime \prime}(\infty)=\sum_{i=1}^{m-2} \alpha_{i} u^{\prime \prime}\left(\xi_{i}\right),
\end{array}\right.
$$

coupled with the resonance condition

$$
\sum_{i=1}^{m-2} \alpha_{i}=1
$$

The techniques used in [6] are basically the same as in [12] and, again, the authors are able to find a solution which could be unbounded. On the other hand, they allow the nonlinearity $f$ to depend on all the derivatives up to the highest possible order but, to do that, they asked for the following quite restrictive condition on the nonlinearity:

$\left(H_{0}\right) f:[0, \infty) \times \mathbb{R}^{3} \rightarrow \mathbb{R}$ is $s^{2}$-Carathéodory, that is,

(i) $f(\cdot, u, v, w)$ is measurable for each $(u, v, w)$ fixed.

(ii) $f(t, \cdot, \cdot, \cdot)$ is continuous for a.e. $t \in[0, \infty)$.

(iii) For each $r>0$ there exists $\psi_{r} \in L^{1}[0, \infty)$ with $t \psi_{r}, t^{2} \psi_{r} \in L^{1}[0, \infty)$ such that

$$
|f(t, u, v, w)| \leq \psi_{r}(t), \quad \forall(u, v, w) \in(-r, r) \times(-r, r) \times(-r, r) \text {, a.e. } t \in[0, \infty) .
$$


Here, we must point out that, although in this paper we work with the second-order problem, the same techniques could be applied to the third-order problem. In this sense, we allow the nonlinearity $f$ to depend on all the derivatives up to the highest possible order but using either hypothesis $\left(H_{1}\right)$ or $\left(H_{2}\right)$ instead of $\left(H_{0}\right)$. This way, our hypotheses regarding the nonlinearity are clearly much less restrictive than $\left(H_{0}\right)$ and our method develops a different approach complementing that in [6].

We would also like to mention that our technique of modifying the problem, in order to obtain another with a related Green's function in $L^{1}[0, \infty) \cap L^{\infty}[0, \infty)$, is also applicable to problems without resonance. Thus, if we used this idea in problems like

$$
\left\{\begin{array}{l}
u^{(4)}(t)+k u(t)=f\left(t, u(t), u^{\prime}(t), u^{\prime \prime}(t), u^{\prime \prime \prime}(t)\right), \quad t \in \mathbb{R} \\
u( \pm \infty)=0, \quad u^{\prime}( \pm \infty)=0
\end{array}\right.
$$

considered in [19], we could extend the results in that reference to nonlinearities satisfying $\left(H_{2}\right)$ instead of $\left(H_{1}\right)$. The same could be said about [18].

The paper is divided into several sections: In Sect. 2, we construct an auxiliary differential problem whose solutions are the same as those of problem (1). In Sect. 3, this auxiliary problem is transformed into an integral one, for which some bounded solutions are found. These solutions are showed to be solutions of the original problem. Finally, Sect. 4 includes an example.

\section{Preliminaries}

We will construct now a modified problem, which will be shown to be equivalent to (1), for which it is possible to construct the related Green's function.

Indeed, consider the modified problem

$$
\left\{\begin{array}{l}
u^{\prime \prime}(t)+k u^{\prime}(t)+M u(t)=0, \quad t \in[0, \infty), \\
u(0)=0, \quad u^{\prime}(\infty)=\sum_{i=1}^{m-1} \alpha_{i} u^{\prime}\left(\xi_{i}\right)
\end{array}\right.
$$

where $k$ and $M$ are positive numbers such that $k^{2}-4 M<0$ and

$$
\sum_{i=1}^{m-1} \alpha_{i} e^{-\frac{k \xi_{i}}{2}}\left(-\frac{k}{2} \sin \left(\gamma \xi_{i}\right)+\gamma \cos \left(\gamma \xi_{i}\right)\right) \neq 0
$$

with $\gamma=\frac{\sqrt{4 M-k^{2}}}{2}$.

If we denote

$$
h_{l}(s)=\frac{\sum_{i=l}^{m-1} \alpha_{i} e^{-\frac{k \xi_{i}}{2}}\left(-\frac{k}{2} \sin \left(\gamma\left(s-\xi_{i}\right)\right)+\gamma \cos \left(\gamma\left(s-\xi_{i}\right)\right)\right)}{\sum_{i=1}^{m-1} \alpha_{i} e^{-\frac{k \xi_{i}}{2}}\left(-\frac{k}{2} \sin \left(\gamma \xi_{i}\right)+\gamma \cos \left(\gamma \xi_{i}\right)\right)}
$$

then the Green's function related to problem (4) is given by the following expression:

$$
G(t, s)=\frac{1}{\gamma} e^{-\frac{k(t+s)}{2}} \begin{cases}-\sin (\gamma t) h_{l}(s), & 0 \leq t \leq s, \xi_{l-1} \leq s<\xi_{l} \\ 0, & 0 \leq t \leq s, \xi_{m-1} \leq s \\ -\sin (\gamma t) h_{l}(s)+\sin (\gamma(s-t)), & 0 \leq s<t, \xi_{l-1} \leq s<\xi_{l} \\ \sin (\gamma(s-t)), & 0 \leq s<t, \xi_{m-1} \leq s\end{cases}
$$


The previous expression has been obtained by some explicit calculations, which will be detailed next. First of all, from the properties of the Green's function (see [4] for the details), we know that it must have the following form:

$$
G(t, s)=e^{-\frac{k}{2} t} \begin{cases}c 1(s) \cos (\gamma t)+c 2(s) \sin (\gamma t), & 0 \leq t \leq s \\ c 3(s) \cos (\gamma t)+c 4(s) \sin (\gamma t), & 0 \leq s<t\end{cases}
$$

for certain functions $c 1, \ldots, c 4$. Moreover, as it must satisfy the boundary conditions, $u(0)=0$ implies that $c 1 \equiv 0$.

Next, from the fact that

$$
\lim _{s \rightarrow t^{-}} \frac{\partial G}{\partial t}(t, s)=\lim _{s \rightarrow t^{+}} \frac{\partial G}{\partial t}(t, s)
$$

and

$$
\lim _{s \rightarrow t^{-}} G(t, s)=\lim _{s \rightarrow t^{+}} G(t, s)+1,
$$

we obtain

$$
c 4(s)=c 2(s)+\frac{1}{\gamma} e^{\frac{k}{2} s} \cos (\gamma s)
$$

and

$$
c 3(s)=-\frac{1}{\gamma} e^{\frac{k}{2} s} \sin (\gamma s) .
$$

Finally, from the condition $u^{\prime}(\infty)=\sum_{i=1}^{m-1} \alpha_{i} u^{\prime}\left(\xi_{i}\right)$, we deduce that

$$
c 2(s)= \begin{cases}-\frac{1}{\gamma} e^{-\frac{k}{2} s} h_{l}(s), & \xi_{l-1} \leq s<\xi_{l}, \\ 0, & \xi_{m-1} \leq s .\end{cases}
$$

The first derivative of the Green's function is given by

$$
\frac{\partial G}{\partial t}(t, s)=\frac{1}{\gamma} e^{-\frac{k(t+s)}{2}} \begin{cases}\left(\frac{k}{2} \sin (\gamma t)-\gamma \cos (\gamma t)\right) h_{l}(s), & 0 \leq t \leq s, \xi_{l-1} \leq s<\xi_{l} \\ 0, & 0 \leq t \leq s, \xi_{m-1} \leq s \\ \left(\frac{k}{2} \sin (\gamma t)-\gamma \cos (\gamma t)\right) h_{l}(s) & \\ -\frac{k}{2} \sin (\gamma(s-t))-\cos (\gamma(s-t)), & 0 \leq s<t, \xi_{l-1} \leq s<\xi_{l} \\ -\frac{k}{2} \sin (\gamma(s-t))-\cos (\gamma(s-t)), & 0 \leq s<t, \xi_{m-1} \leq s .\end{cases}
$$

Remark 1 It is easy to see that there exist two positive constants, $C_{1}$ and $C_{2}$, such that

$$
|G(t, s)| \leq C_{1} e^{-\frac{k(t+s)}{2}}
$$

and

$$
\left|\frac{\partial G}{\partial t}(t, s)\right| \leq C_{2} e^{-\frac{k(t+s)}{2}},
$$

for all $(t, s) \in[0, \infty) \times[0, \infty)$. 
As a consequence, it is clear that both $G(t, \cdot)$ and $\frac{\partial G}{\partial t}(t, \cdot)$ belong to $L^{1}[0, \infty) \cap L^{\infty}[0, \infty)$ for all $t \in[0, \infty)$.

Let us consider

$$
X=\left\{u \in \mathcal{C}^{1}[0, \infty): \sup _{t \in[0, \infty)}|u(t)|<\infty, \sup _{t \in[0, \infty)}\left|u^{\prime}(t)\right|<\infty\right\}
$$

equipped with the norm

$$
\|u\|=\max \left\{\|u\|_{\infty},\left\|u^{\prime}\right\|_{\infty}\right\}
$$

where $\|v\|_{\infty}=\sup _{t \in[0, \infty)}|v(t)|$. It is easy to prove that $(X,\|\cdot\|)$ is a Banach space.

To deal with the lack of compactness of the set $X$, we will use the following result:

Theorem 2 ([5]) Let $Y$ be a Banach space and $\mathcal{C}(\mathbb{R}, Y)$ the space of all bounded continuous functions $x: \mathbb{R} \rightarrow Y$. For a set $D \subset \mathcal{C}(\mathbb{R}, Y)$ to be relatively compact, it is necessary and sufficient that:

1. D is uniformly bounded;

2. Functions from $D$ are equicontinuous on every compact subinterval of $[0, \infty)$;

3. Functions from $D$ are equiconvergent at $\infty$, that is, given $\varepsilon>0$, there exists $T>0$ such that for all $t \geq T$, we have that

$$
\left\|x(t)-\lim _{t \rightarrow \infty} x(t)\right\|<\varepsilon .
$$

To prove the existence of solutions, we will consider two different results. First of all, we will use the very well-known Schauder's fixed point theorem:

Theorem 3 ([26]) Let $Y$ be a nonempty, closed, bounded and convex subset of a Banach space $X$, and suppose that $P: Y \rightarrow Y$ is a compact operator. Then $P$ has at least one fixed point in $Y$.

On the other hand, we will also give a result to prove the existence of solutions based on the lower and upper solutions technique. To do that, we need to introduce the following definition:

Definition 4 A function $\alpha \in X$ is said to be a lower solution of problem (1) if

$$
\left\{\begin{array}{l}
\alpha^{\prime \prime}(t) \geq f\left(t, \alpha(t), \alpha^{\prime}(t)\right), \quad t \in[0, \infty) \\
\alpha(0) \leq 0, \quad \alpha^{\prime}(\infty) \geq \sum_{i=1}^{m-1} \alpha_{i} \alpha^{\prime}\left(\xi_{i}\right)
\end{array}\right.
$$

A function $\beta \in X$ is said to be an upper solution of (1) if the reversed inequalities hold.

\section{Main results}

Consider the following integral operator $T: X \rightarrow X$ defined by

$$
T u(t)=\int_{0}^{\infty} G(t, s)\left(f\left(s, u(s), u^{\prime}(s)\right)+k u^{\prime}(s)+M u(s)\right) \mathrm{d} s
$$

It is clear that solutions of problem (1) are fixed points of operator $T$. 
Moreover, we will assume that at least one of the two following conditions holds:

$\left(H_{1}\right)$ The nonlinearity $f:[0, \infty) \times \mathbb{R}^{2} \rightarrow \mathbb{R}$ satisfies $L^{1}$-Carathéodory condition, that is,

(i) $f(\cdot, u, v)$ is measurable for each $(u, v)$ fixed.

(ii) $f(t, \cdot, \cdot)$ is continuous for a.e. $t \in[0, \infty)$.

(iii) For each $r>0$ there exists $\varphi_{r} \in L^{1}[0, \infty)$ such that

$$
|f(t, u, v)| \leq \varphi_{r}(t), \quad \forall(u, v) \in(-r, r) \times(-r, r) \text {, a.e. } t \in[0, \infty)
$$

$\left(H_{2}\right)$ The nonlinearity $f:[0, \infty) \times \mathbb{R}^{2} \rightarrow \mathbb{R}$ satisfies $L^{\infty}$-Carathéodory condition, that is,

(i) $f(\cdot, u, v)$ is measurable for each $(u, v)$ fixed.

(ii) $f(t, \cdot, \cdot)$ is continuous for a.e. $t \in[0, \infty)$.

(iii) For each $r>0$ there exists $\phi_{r} \in L^{\infty}[0, \infty)$ such that

$$
|f(t, u, v)| \leq \phi_{r}(t), \quad \forall(u, v) \in(-r, r) \times(-r, r) \text {, a.e. } t \in[0, \infty)
$$

Under one of these conditions, we will be able to prove the following result.

Lemma 5 Assume that either $\left(H_{1}\right)$ or $\left(H_{2}\right)$ holds. Then operator $T$ defined in (5) is completely continuous.

Proof The proof will be divided into several steps.

Step 1: $T$ is well-defined in $X$.

Given an arbitrary $u \in X$, we will prove that $T u \in X$.

First, we will make the proof in case hypothesis $\left(H_{1}\right)$ holds. If $u \in X$, then there exists some $r>0$ such that $\|u\|<r$. Therefore, it holds that

$$
\begin{aligned}
|T u(t)| & =\left|\int_{0}^{\infty} G(t, s)\left(f\left(s, u(s), u^{\prime}(s)\right)+k u^{\prime}(s)+M u(s)\right) \mathrm{d} s\right| \\
& \leq \int_{0}^{\infty}|G(t, s)|\left(\left|f\left(s, u(s), u^{\prime}(s)\right)\right|+k\left|u^{\prime}(s)\right|+M|u(s)|\right) \mathrm{d} s \\
& \leq \int_{0}^{\infty}|G(t, s)|\left(\varphi_{r}(s)+(k+M) r\right) \mathrm{d} s \\
& \leq \int_{0}^{\infty} C_{1} e^{-\frac{k(t+s)}{2}}\left(\varphi_{r}(s)+(k+M) r\right) \mathrm{d} s \\
& =C_{1} e^{-\frac{k t}{2}}\left(\int_{0}^{\infty} e^{-\frac{k s}{2}} \varphi_{r}(s) \mathrm{d} s+\frac{2}{k}(k+M) r\right) \\
& =C_{1} e^{-\frac{k t}{2}}\left(\int_{0}^{\infty} e^{-\frac{k s}{2}} \varphi_{r}(s) \mathrm{d} s+\left(2+\frac{2 M}{k}\right) r\right)
\end{aligned}
$$

and, analogously,

$$
\begin{aligned}
\left|(T u)^{\prime}(t)\right| & =\left|\int_{0}^{\infty} \frac{\partial G}{\partial t}(t, s)\left(f\left(s, u(s), u^{\prime}(s)\right)+k u^{\prime}(s)+M u(s)\right) \mathrm{d} s\right| \\
& \leq \int_{0}^{\infty}\left|\frac{\partial G}{\partial t}(t, s)\right|\left(\left|f\left(s, u(s), u^{\prime}(s)\right)\right|+k\left|u^{\prime}(s)\right|+M|u(s)|\right) \mathrm{d} s \\
& \leq \int_{0}^{\infty}\left|\frac{\partial G}{\partial t}(t, s)\right|\left(\varphi_{r}(s)+(k+M) r\right) \mathrm{d} s
\end{aligned}
$$




$$
\begin{aligned}
& \leq \int_{0}^{\infty} C_{2} e^{-\frac{k(t+s)}{2}}\left(\varphi_{r}(s)+(k+M) r\right) \mathrm{d} s \\
& =C_{2} e^{-\frac{k t}{2}}\left(\int_{0}^{\infty} e^{-\frac{k s}{2}} \varphi_{r}(s) \mathrm{d} s+\left(2+\frac{2 M}{k}\right) r\right) .
\end{aligned}
$$

Now, since $\varphi_{r} \in L^{1}[0, \infty)$ and $e^{-\frac{k s}{2}} \in L^{\infty}[0, \infty)$, it holds that $\varphi_{r}(s) e^{-\frac{k s}{2}} \in L^{1}[0, \infty)$. Thus, it is clear that

$$
\sup _{t \in[0, \infty)}|T u(t)|<\infty \text { and } \sup _{t \in[0, \infty)}\left|(T u)^{\prime}(t)\right|<\infty
$$

that is, $T u \in X$.

On the other hand, if $\left(H_{2}\right)$ holds instead of $\left(H_{1}\right)$, following similar steps as in the previous case, we obtain the following upper bounds:

$$
|T u(t)| \leq C_{1} e^{-\frac{k t}{2}}\left(\int_{0}^{\infty} e^{-\frac{k s}{2}} \phi_{r}(s) \mathrm{d} s+\left(2+\frac{2 M}{k}\right) r\right)
$$

and

$$
\left|(T u)^{\prime}(t)\right| \leq C_{2} e^{-\frac{k t}{2}}\left(\int_{0}^{\infty} e^{-\frac{k s}{2}} \phi_{r}(s) \mathrm{d} s+\left(2+\frac{2 M}{k}\right) r\right) .
$$

In this case $\phi_{r} \in L^{\infty}[0, \infty)$ and, since $e^{-\frac{k s}{2}} \in L^{1}[0, \infty)$, we obtain that $\phi_{r}(s) e^{-\frac{k s}{2}} \in L^{1}[0, \infty)$. Therefore we conclude again that $T u \in X$.

Step 2: $T$ is a continuous operator.

We will detail the proof for the case in which $\left(H_{1}\right)$ holds. For $\left(H_{2}\right)$ the proof will be analogous, with the obvious changes, as done in Step 1.

Consider a sequence $\left\{u_{n}\right\}_{n \in \mathbb{N}}$ and assume that it converges to $u$ in $X$, that is,

$$
\lim _{n \rightarrow \infty} u_{n}(t)=u(t) \quad \text { and } \quad \lim _{n \rightarrow \infty} u_{n}^{\prime}(t)=u^{\prime}(t) \text { for all } t \in[0, \infty)
$$

Then, since $f(t, \cdot, \cdot)$ is continuous for a.e. $t \in[0, \infty)$, we deduce that

$$
\lim _{n \rightarrow \infty} f\left(s, u_{n}(s), u_{n}^{\prime}(s)\right)=f\left(s, u(s), u^{\prime}(s)\right) \quad \text { for a.e. } s \in[0, \infty)
$$

Let us see that $\left\{T u_{n}\right\}_{n \in \mathbb{N}}$ converges to $T u$.

Since $\left\{u_{n}\right\}_{n \in \mathbb{N}}$ is convergent in $X$, there exists some $r>0$ such that $\left\|u_{n}\right\|<r$ for all $n \in \mathbb{N}$. Now, if $\left(H_{1}\right)$ holds,

$$
\begin{aligned}
\left|T u_{n}(t)-T u(t)\right| \leq & \int_{0}^{\infty}|G(t, s)| f\left(s, u_{n}(s), u_{n}^{\prime}(s)\right)-f\left(s, u(s), u^{\prime}(s)\right) \mid \mathrm{d} s \\
& +\int_{0}^{\infty}|G(t, s)|\left(k\left|u_{n}^{\prime}(s)-u^{\prime}(s)\right|+M\left|u_{n}(s)-u(s)\right|\right) \mathrm{d} s \\
\leq & C_{1} \int_{0}^{\infty} e^{-\frac{k(t+s)}{2}}\left|f\left(s, u_{n}(s), u_{n}^{\prime}(s)\right)-f\left(s, u(s), u^{\prime}(s)\right)\right| \mathrm{d} s \\
& +C_{1} \int_{0}^{\infty} e^{-\frac{k(t+s)}{2}}\left(k\left|u_{n}^{\prime}(s)-u^{\prime}(s)\right|+M\left|u_{n}(s)-u(s)\right|\right) \mathrm{d} s \\
\leq & C_{1} \int_{0}^{\infty} e^{-\frac{k s}{2}}\left(2 \varphi_{r}(s)+2(k+M) r\right) \mathrm{d} s<\infty .
\end{aligned}
$$


Then, we deduce from Lebesgue's Dominated Convergence Theorem that

$$
\begin{aligned}
\lim _{n \rightarrow \infty}\left\|T u_{n}-T u\right\|_{\infty} \leq & \lim _{n \rightarrow \infty} C_{1} \int_{0}^{\infty} e^{-\frac{k s}{2}}\left|f\left(s, u_{n}(s), u_{n}^{\prime}(s)\right)-f\left(s, u(s), u^{\prime}(s)\right)\right| \mathrm{d} s \\
& +\lim _{n \rightarrow \infty} C_{1} \int_{0}^{\infty} e^{-\frac{k s}{2}}\left(k\left|u_{n}^{\prime}(s)-u^{\prime}(s)\right|+M\left|u_{n}(s)-u(s)\right|\right) \mathrm{d} s \\
= & C_{1} \int_{0}^{\infty} \lim _{n \rightarrow \infty} e^{-\frac{k s}{2}}\left|f\left(s, u_{n}(s), u_{n}^{\prime}(s)\right)-f\left(s, u(s), u^{\prime}(s)\right)\right| \mathrm{d} s \\
& +C_{1} \int_{0}^{\infty} \lim _{n \rightarrow \infty} e^{-\frac{k s}{2}}\left(k\left|u_{n}^{\prime}(s)-u^{\prime}(s)\right|+M\left|u_{n}(s)-u(s)\right|\right) \mathrm{d} s \\
= & 0 .
\end{aligned}
$$

Analogously, we get that

$$
\begin{aligned}
\lim _{n \rightarrow \infty}\left\|\left(T u_{n}\right)^{\prime}-(T u)^{\prime}\right\|_{\infty} \leq & C_{2} \int_{0}^{\infty} \lim _{n \rightarrow \infty} e^{-\frac{k s}{2}}\left|f\left(s, u_{n}(s), u_{n}^{\prime}(s)\right)-f\left(s, u(s), u^{\prime}(s)\right)\right| \mathrm{d} s \\
& +C_{2} \int_{0}^{\infty} \lim _{n \rightarrow \infty} e^{-\frac{k s}{2}}\left(k\left|u_{n}^{\prime}(s)-u^{\prime}(s)\right|+M\left|u_{n}(s)-u(s)\right|\right) \mathrm{d} s
\end{aligned}
$$

$=0$.

Thus, $\left\{T u_{n}\right\}_{n \in \mathbb{N}}$ converges to $T u$ in $X$.

Step 3: $T$ is compact.

Again, we will make the proof only for the case in which $\left(H_{1}\right)$ holds, the other case being analogous.

Let $B$ be a bounded subset of $X$, that is, there exists some $r>0$ such that $\|u\|<r$, for all $u \in B$. Let us see that $T(B)$ is relatively compact in $X$.

(i) $T(B)$ is uniformly bounded.

If $u \in B$, then, for $t \in[0, \infty)$,

$$
\begin{aligned}
|T u(t)| & \leq C_{1} e^{-\frac{k t}{2}}\left(\int_{0}^{\infty} e^{-\frac{k s}{2}} \varphi_{r}(s) \mathrm{d} s+\left(2+\frac{2 M}{k}\right) r\right) \\
& \leq C_{1}\left(\int_{0}^{\infty} e^{-\frac{k s}{2}} \varphi_{r}(s) \mathrm{d} s+\left(2+\frac{2 M}{k}\right) r\right)=: M_{1}>0
\end{aligned}
$$

and

$$
\begin{aligned}
\left|(T u)^{\prime}(t)\right| & \leq C_{2} e^{-\frac{k t}{2}}\left(\int_{0}^{\infty} e^{-\frac{k s}{2}} \varphi_{r}(s) \mathrm{d} s+\left(2+\frac{2 M}{k}\right) r\right) \\
& \leq C_{2}\left(\int_{0}^{\infty} e^{-\frac{k s}{2}} \varphi_{r}(s) \mathrm{d} s+\left(2+\frac{2 M}{k}\right) r\right)=: M_{2}>0 .
\end{aligned}
$$

Thus,

$$
\|T u\| \leq \max \left\{M_{1}, M_{2}\right\}
$$

for all $u \in B$, that is, $T(B)$ is uniformly bounded. 
(ii) $T(B)$ is equicontinuous.

Let $t_{1}, t_{2} \in[0, L]$ for some $L>0$ and assume that $t_{1}>t_{2}$. Then,

$$
\begin{aligned}
\left|T u\left(t_{1}\right)-T u\left(t_{2}\right)\right| & \\
\leq & \int_{0}^{\infty}\left|G\left(t_{1}, s\right)-G\left(t_{2}, s\right)\right|\left(\left|f\left(s, u(s), u^{\prime}(s)\right)\right|+k\left|u^{\prime}(s)\right|+M|u(s)|\right) \mathrm{d} s \\
\leq & \int_{0}^{\infty}\left|G\left(t_{1}, s\right)-G\left(t_{2}, s\right)\right|\left(\varphi_{r}(s)+(k+M) r\right) \mathrm{d} s \\
= & \int_{0}^{t_{2}}\left|G\left(t_{1}, s\right)-G\left(t_{2}, s\right)\right|\left(\varphi_{r}(s)+(k+M) r\right) \mathrm{d} s \\
& +\int_{t_{2}}^{t_{1}}\left|G\left(t_{1}, s\right)-G\left(t_{2}, s\right)\right|\left(\varphi_{r}(s)+(k+M) r\right) \mathrm{d} s \\
& +\int_{t_{1}}^{\infty}\left|G\left(t_{1}, s\right)-G\left(t_{2}, s\right)\right|\left(\varphi_{r}(s)+(k+M) r\right) \mathrm{d} s .
\end{aligned}
$$

We will find some suitable upper bounds for the difference $\left|G\left(t_{1}, s\right)-G\left(t_{2}, s\right)\right|$. For $0 \leq t_{2}<t_{1} \leq s$, we have two possibilities:

- If $\xi_{l-1} \leq s<\xi_{l}$ for some $2 \leq l \leq m-1$, then

$$
\left|G\left(t_{1}, s\right)-G\left(t_{2}, s\right)\right|=\frac{1}{\gamma}\left|h_{l}(s)\right| e^{-\frac{k s}{2}}\left|-e^{-\frac{k t_{1}}{2}} \sin \left(\gamma t_{1}\right)+e^{-\frac{k t_{2}}{2}} \sin \left(\gamma t_{2}\right)\right| .
$$

- If $s \geq \xi_{m-1}$, then

$$
\left|G\left(t_{1}, s\right)-G\left(t_{2}, s\right)\right|=0
$$

On the other hand, for $0 \leq s \leq t_{2}<t_{1}$ :

- If $\xi_{l-1} \leq s<\xi_{l}$ for some $2 \leq l \leq m-1$, then

$$
\begin{aligned}
\left|G\left(t_{1}, s\right)-G\left(t_{2}, s\right)\right| \leq & \frac{1}{\gamma}\left|h_{l}(s)\right| e^{-\frac{k s}{2}}\left|-e^{-\frac{k t_{1}}{2}} \sin \left(\gamma t_{1}\right)+e^{-\frac{k t_{2}}{2}} \sin \left(\gamma t_{2}\right)\right| \\
& +\frac{1}{\gamma} e^{-\frac{k s}{2}}\left|e^{-\frac{k t_{1}}{2}} \sin \left(\gamma\left(s-t_{1}\right)\right)-e^{-\frac{k t_{2}}{2}} \sin \left(\gamma\left(s-t_{2}\right)\right)\right| .
\end{aligned}
$$

Last term in the previous sum can be upper-bounded as follows:

$$
\begin{aligned}
\mid e^{-\frac{k t_{1}}{2}} & \sin \left(\gamma\left(s-t_{1}\right)\right)-e^{-\frac{k t_{2}}{2}} \sin \left(\gamma\left(s-t_{2}\right)\right) \mid \\
= & |\sin (\gamma s)|\left|e^{-\frac{k t_{1}}{2}} \cos \left(\gamma t_{1}\right)-e^{-\frac{k t_{2}}{2}} \cos \left(\gamma t_{2}\right)\right| \\
& +|\cos (\gamma s)|\left|-e^{-\frac{k t_{1}}{2}} \sin \left(\gamma t_{1}\right)+e^{-\frac{k t_{2}}{2}} \sin \left(\gamma t_{2}\right)\right| \\
\leq & \left|e^{-\frac{k t_{1}}{2}} \cos \left(\gamma t_{1}\right)-e^{-\frac{k t_{2}}{2}} \cos \left(\gamma t_{2}\right)\right| \\
& +\left|-e^{-\frac{k t_{1}}{2}} \sin \left(\gamma t_{1}\right)+e^{-\frac{k t_{2}}{2}} \sin \left(\gamma t_{2}\right)\right| .
\end{aligned}
$$


As a consequence,

$$
\begin{aligned}
\left|G\left(t_{1}, s\right)-G\left(t_{2}, s\right)\right| \leq & \frac{1}{\gamma}\left(\left|h_{l}(s)\right|+1\right) e^{-\frac{k s}{2}}\left|-e^{-\frac{k t_{1}}{2}} \sin \left(\gamma t_{1}\right)+e^{-\frac{k t_{2}}{2}} \sin \left(\gamma t_{2}\right)\right| \\
& +\frac{1}{\gamma} e^{-\frac{k s}{2}}\left|e^{-\frac{k t_{1}}{2}} \cos \left(\gamma t_{1}\right)-e^{-\frac{k t_{2}}{2}} \cos \left(\gamma t_{2}\right)\right| .
\end{aligned}
$$

- If $s \geq \xi_{m-1}$, then

$$
\begin{aligned}
\left|G\left(t_{1}, s\right)-G\left(t_{2}, s\right)\right|= & \frac{1}{\gamma} e^{-\frac{k s}{2}}\left|e^{-\frac{k t_{1}}{2}} \sin \left(\gamma\left(s-t_{1}\right)\right)-e^{-\frac{k t_{2}}{2}} \sin \left(\gamma\left(s-t_{2}\right)\right)\right| \\
\leq & \frac{1}{\gamma} e^{-\frac{k s}{2}}\left|e^{-\frac{k t_{1}}{2}} \cos \left(\gamma t_{1}\right)-e^{-\frac{k t_{2}}{2}} \cos \left(\gamma t_{2}\right)\right| \\
& +\frac{1}{\gamma} e^{-\frac{k s}{2}}\left|-e^{-\frac{k t_{1}}{2}} \sin \left(\gamma t_{1}\right)+e^{-\frac{k t_{2}}{2}} \sin \left(\gamma t_{2}\right)\right| .
\end{aligned}
$$

Therefore, we can affirm that for a given $\varepsilon>0$ there exists some $\delta>0$ such that if $\left|t_{1}-t_{2}\right|<\delta$ then, for $s \in\left[0, t_{2}\right) \cup\left(t_{1}, \infty\right)$, it holds that

$$
\left|G\left(t_{1}, s\right)-G\left(t_{2}, s\right)\right| \leq \varepsilon e^{-\frac{k s}{2}}
$$

This implies that the first and third terms of the last part of inequality (8) tend to zero regardless of the function $u \in B$.

On the other hand, for $0 \leq t_{2} \leq s \leq t_{1}$ :

- If $\xi_{l-1} \leq s<\xi_{l}$ for some $2 \leq l \leq m-1$, then

$$
\begin{aligned}
\left|G\left(t_{1}, s\right)-G\left(t_{2}, s\right)\right| \leq & \frac{1}{\gamma} e^{-\frac{k s}{2}}\left|h_{l}(s)\right|\left|-e^{-\frac{k t_{1}}{2}} \sin \left(\gamma t_{1}\right)+e^{-\frac{k t_{2}}{2}} \sin \left(\gamma t_{2}\right)\right| \\
& +\frac{1}{\gamma} e^{-\frac{k s}{2}}\left|e^{-\frac{k t_{1}}{2}} \sin \left(\gamma\left(s-t_{1}\right)\right)\right| .
\end{aligned}
$$

- If $s \geq \xi_{m-1}$, then

$$
\left|G\left(t_{1}, s\right)-G\left(t_{2}, s\right)\right|=\frac{1}{\gamma} e^{-\frac{k s}{2}}\left|e^{-\frac{k t_{1}}{2}} \sin \left(\gamma\left(s-t_{1}\right)\right)\right| .
$$

Thus, when $s \in\left[t_{2}, t_{1}\right]$, it holds that

$$
\left|G\left(t_{1}, s\right)-G\left(t_{2}, s\right)\right| \leq C e^{-\frac{k s}{2}},
$$

for some constant $C$. This implies that $\left|G\left(t_{1}, \cdot\right)-G\left(t_{2}, \cdot\right)\right|\left(\varphi_{r}(\cdot)+(k+M) r\right) \in L^{1}\left[t_{1}, t_{2}\right]$ for any $t_{1}, t_{2} \in[0, \infty)$. Then it is clear that

$$
\int_{t_{2}}^{t_{1}}\left|G\left(t_{1}, s\right)-G\left(t_{2}, s\right)\right|\left(\varphi_{r}(s)+(k+M) r\right) \mathrm{d} s \underset{t_{1} \rightarrow t_{2}}{\longrightarrow} 0
$$

regardless of the function $u \in B$.

Thus we conclude that given $\varepsilon>0$ there exists $\delta(\varepsilon)>0$ such that if $\left|t_{1}-t_{2}\right|<\delta$, then $\left|T u\left(t_{1}\right)-T u\left(t_{2}\right)\right|<\varepsilon$ for all $u \in B$. 
In a completely analogous way, finding suitable upper bounds for $\left|\frac{\partial G}{\partial t}\left(t_{1}, s\right)-\frac{\partial G}{\partial t}\left(t_{2}, s\right)\right|$, it is possible to prove that given $\varepsilon>0$ there exists $\delta(\varepsilon)>0$ such that if $\left|t_{1}-t_{2}\right|<\delta$, then $\left|(T u)^{\prime}\left(t_{1}\right)-(T u)^{\prime}\left(t_{2}\right)\right|<\varepsilon$ for all $u \in B$.

Therefore, $T(B)$ is equicontinuous.

(iii) $T(B)$ is equiconvergent at $\infty$.

Given $u \in B$, it holds that

$$
\begin{aligned}
\left|T u(t)-\lim _{t \rightarrow \infty} T u(t)\right| \leq & C_{1} e^{-\frac{k t}{2}}\left(\int_{0}^{\infty} e^{-\frac{k s}{2}} \varphi_{r}(s) \mathrm{d} s+\left(2+\frac{2 M}{k}\right) r\right) \\
& +\lim _{t \rightarrow \infty} C_{1} e^{-\frac{k t}{2}}\left(\int_{0}^{\infty} e^{-\frac{k s}{2}} \varphi_{r}(s) \mathrm{d} s+\left(2+\frac{2 M}{k}\right) r\right) \\
\leq & C_{1} e^{-\frac{k t}{2}}\left(\int_{0}^{\infty} e^{-\frac{k s}{2}} \varphi_{r}(s) \mathrm{d} s+\left(2+\frac{2 M}{k}\right) r\right) \underset{t \rightarrow \infty}{\longrightarrow} 0
\end{aligned}
$$

and

$$
\begin{aligned}
\left|(T u)^{\prime}(t)-\lim _{t \rightarrow \infty}(T u)^{\prime}(t)\right| \leq & C_{2} e^{-\frac{k t}{2}}\left(\int_{0}^{\infty} e^{-\frac{k s}{2}} \varphi_{r}(s) \mathrm{d} s+\left(2+\frac{2 M}{k}\right) r\right) \\
& +\lim _{t \rightarrow \infty} C_{2} e^{-\frac{k t}{2}}\left(\int_{0}^{\infty} e^{-\frac{k s}{2}} \varphi_{r}(s) \mathrm{d} s+\left(2+\frac{2 M}{k}\right) r\right) \\
\leq & C_{2} e^{-\frac{k t}{2}}\left(\int_{0}^{\infty} e^{-\frac{k s}{2}} \varphi_{r}(s) \mathrm{d} s+\left(2+\frac{2 M}{k}\right) r\right) \underset{t \rightarrow \infty}{\longrightarrow} 0,
\end{aligned}
$$

that is, $T B$ is equiconvergent at $\infty$.

Therefore, from Theorem 2, we conclude that $T(B)$ is relatively compact in $X$. Now we will see our existence results.

Theorem 6 Let $f:[0, \infty) \times \mathbb{R}^{2} \rightarrow \mathbb{R}$ be such that there exists $t_{0} \in[0, \infty)$ for which $f\left(t_{0}, 0,0\right) \neq 0$. Moreover, suppose that, for $C_{1}$ and $C_{2}$ given in Remark 1 , either

- $\left(H_{1}\right)$ holds and, moreover, there exists some $R>0$ such that

$$
\begin{aligned}
& \max \left\{C_{1}, C_{2}\right\} \max \left\{\sup _{t>\xi_{m-1}} e^{-\frac{k t}{2}} \int_{0}^{t} e^{-\frac{k s}{2}} \varphi_{R}(s) \mathrm{d} s, \int_{0}^{\xi_{m-1}} e^{-\frac{k s}{2}} \varphi_{R}(s) \mathrm{d} s\right\} \\
& +\max \left\{C_{1}, C_{2}\right\} \max \left\{\frac{1}{2}, 2\left(1-e^{-\frac{k \xi_{m-1}}{2}}\right)\right\}\left(1+\frac{M}{k}\right) R<R,
\end{aligned}
$$

or

- $\left(\mathrm{H}_{2}\right)$ holds and, moreover, there is $\mathrm{R}>0$ such that

$$
\begin{aligned}
& \max \left\{C_{1}, C_{2}\right\} \max \left\{\sup _{t>\xi_{m-1}} e^{-\frac{k t}{2}} \int_{0}^{t} e^{-\frac{k s}{2}} \phi_{R}(s) \mathrm{d} s, \int_{0}^{\xi_{m-1}} e^{-\frac{k s}{2}} \phi_{R}(s) \mathrm{d} s\right\} \\
& +\max \left\{C_{1}, C_{2}\right\} \max \left\{\frac{1}{2}, 2\left(1-e^{-\frac{k \xi_{m-1}}{2}}\right)\right\}\left(1+\frac{M}{k}\right) R<R .
\end{aligned}
$$

Then problem (1) has at least one nontrivial solution. 
Proof We will prove the first case, the second being analogous.

Consider

$$
D=\{u \in X:\|u\|<R\} .
$$

If $u \in D$ then

$$
|T u(t)| \leq \int_{0}^{\infty}|G(t, s)|\left(\varphi_{R}(s)+(k+M) R\right) \mathrm{d} s, \forall t \in[0, \infty),
$$

and, since $G(t, s)=0$ for $s \geq \max \left\{t, \xi_{m-1}\right\}$,

$$
|T u(t)| \leq \int_{0}^{\max \left\{t, \xi_{m-1}\right\}}|G(t, s)|\left(\varphi_{R}(s)+(k+M) R\right) \mathrm{d} s, \forall t \in[0, \infty) .
$$

If $t>\xi_{m-1}$, the previous expression leads to

$$
\begin{aligned}
|T u(t)| & \leq \int_{0}^{t}|G(t, s)|\left(\varphi_{R}(s)+(k+M) R\right) \mathrm{d} s \\
& \leq C_{1} e^{-\frac{k t}{2}} \int_{0}^{t} e^{-\frac{k s}{2}}\left(\varphi_{R}(s)+(k+M) R\right) \mathrm{d} s \\
& \leq C_{1}\left(e^{-\frac{k t}{2}} \int_{0}^{t} e^{-\frac{k s}{2}} \varphi_{R}(s) \mathrm{d} s+2 e^{-\frac{k t}{2}}\left(1-e^{-\frac{k t}{2}}\right)\left(1+\frac{M}{k}\right) R\right) \\
& \leq C_{1}\left(e^{-\frac{k t}{2}} \int_{0}^{t} e^{-\frac{k s}{2}} \varphi_{R}(s) \mathrm{d} s+\frac{1}{2}\left(1+\frac{M}{k}\right) R\right) .
\end{aligned}
$$

On the other hand, if $t \leq \xi_{m-1}$, we obtain that

$$
\begin{aligned}
|T u(t)| & \leq \int_{0}^{\xi_{m-1}}|G(t, s)|\left(\varphi_{R}(s)+(k+M) R\right) \mathrm{d} s \\
& \leq C_{1} e^{-\frac{k t}{2}} \int_{0}^{\xi_{m-1}} e^{-\frac{k s}{2}}\left(\varphi_{R}(s)+(k+M) R\right) \mathrm{d} s \\
& \leq C_{1}\left(e^{-\frac{k t}{2}} \int_{0}^{\xi_{m-1}} e^{-\frac{k s}{2}} \varphi_{R}(s) \mathrm{d} s+2 e^{-\frac{k t}{2}}\left(1-e^{-\frac{k \xi_{m-1}}{2}}\right)\left(1+\frac{M}{k}\right) R\right) \\
& \leq C_{1}\left(\int_{0}^{\xi_{m-1}} e^{-\frac{k s}{2}} \varphi_{R}(s) \mathrm{d} s+2\left(1-e^{-\frac{k \xi_{m-1}}{2}}\right)\left(1+\frac{M}{k}\right) R\right) .
\end{aligned}
$$

Therefore,

$$
\begin{aligned}
|T u(t)| \leq & C_{1} \max \left\{\sup _{t>\xi_{m-1}} e^{-\frac{k t}{2}} \int_{0}^{t} e^{-\frac{k s}{2}} \varphi_{R}(s) \mathrm{d} s, \int_{0}^{\xi_{m-1}} e^{-\frac{k s}{2}} \varphi_{R}(s) \mathrm{d} s\right\} \\
& +C_{1} \max \left\{\frac{1}{2}, 2\left(1-e^{-\frac{k \xi_{m-1}}{2}}\right)\right\}\left(1+\frac{M}{k}\right) R, \quad \forall t \in[0, \infty) .
\end{aligned}
$$


Analogously, it can be seen that

$$
\begin{aligned}
\left|(T u)^{\prime}(t)\right| \leq & C_{2} \max \left\{\sup _{t>\xi_{m-1}} e^{-\frac{k t}{2}} \int_{0}^{t} e^{-\frac{k s}{2}} \varphi_{R}(s) \mathrm{d} s, \int_{0}^{\xi_{m-1}} e^{-\frac{k s}{2}} \varphi_{R}(s) \mathrm{d} s\right\} \\
& +C_{2} \max \left\{\frac{1}{2}, 2\left(1-e^{-\frac{k \xi_{m-1}}{2}}\right)\right\}\left(1+\frac{M}{k}\right) R, \quad \forall t \in[0, \infty) .
\end{aligned}
$$

Thus, by (9),

$$
\begin{aligned}
\|T u\| \leq & \max \left\{C_{1}, C_{2}\right\} \max \left\{\sup _{t>\xi_{m-1}} e^{-\frac{k t}{2}} \int_{0}^{t} e^{-\frac{k s}{2}} \varphi_{R}(s) \mathrm{d} s, \int_{0}^{\xi_{m-1}} e^{-\frac{k s}{2}} \varphi_{R}(s) \mathrm{d} s\right\} \\
& +\max \left\{C_{1}, C_{2}\right\} \max \left\{\frac{1}{2}, 2\left(1-e^{-\frac{k \xi_{m-1}}{2}}\right)\right\}\left(1+\frac{M}{k}\right) R<R,
\end{aligned}
$$

that is, $T u \in D$.

Therefore, $T D \subset D$ and, from Theorem 3, the operator $T$ has at least one fixed point in $D$, which is a solution of problem (1). Moreover, since there exists at least one value $t_{0} \in[0, \infty)$ for which $f\left(t_{0}, 0,0\right) \neq 0$, this solution cannot be the trivial one.

Now, we will give another existence result based on the lower and upper solutions technique. The proof will follow the lines of [23]. Before formulating the theorem, we will give a lemma that we will use in the proof.

Lemma 7 ([23, Lemma 2]) Let $u \in \mathcal{C}^{1}(I), v, w \in W^{2,1}(I)$ and define

$$
p(t, u(t))= \begin{cases}v(t), & u(t)<v(t) \\ u(t), & v(t) \leq u(t) \leq w(t) \\ w(t), & u(t)>w(t)\end{cases}
$$

Then, the two following properties hold:

1. $\frac{\mathrm{d}}{\mathrm{d} t} p(t, u(t))$ exists for a.e. $t \in I$.

2. If $u, u_{m} \in \mathcal{C}^{1}(I)$ and $u_{m}$ converges to $u$ in $\mathcal{C}^{1}(I)$, then

$$
\lim _{m \rightarrow \infty} \frac{\mathrm{d}}{\mathrm{d} t} p\left(t, u_{m}(t)\right)=\frac{\mathrm{d}}{\mathrm{d} t} p(t, u(t)), \quad \text { a.e. } t \in I
$$

Theorem 8 Let $\alpha, \beta \in X$ be lower and upper solutions of problem (1), respectively, with

$$
\alpha(t) \leq \beta(t), \quad \forall t \in[0, \infty)
$$

and denote

$$
\widetilde{R}=\max \left\{\|\alpha\|_{\infty},\|\beta\|_{\infty},\left\|\alpha^{\prime}\right\|_{\infty},\left\|\beta^{\prime}\right\|_{\infty}\right\}
$$


- $\left(H_{1}\right)$ holds and, moreover, there exists some $R>0$ such that

$$
\begin{aligned}
& \max \left\{C_{1}, C_{2}\right\} \max \left\{\sup _{t>\xi_{m-1}} e^{-\frac{k t}{2}} \int_{0}^{t} e^{-\frac{k s}{2}} \varphi_{\max \{R, \widetilde{R}\}}(s) \mathrm{d} s, \int_{0}^{\xi_{m-1}} e^{-\frac{k s}{2}} \varphi_{\max \{R, \widetilde{R}\}}(s) \mathrm{d} s\right\} \\
& +\max \left\{C_{1}, C_{2}\right\} \max \left\{\frac{1}{2}, 2\left(1-e^{-\frac{k \xi_{m-1}}{2}}\right)\right\}\left(1+\frac{M}{k}\right) R<R,
\end{aligned}
$$

or

- $\left(H_{2}\right)$ holds and, moreover, there exists some $R>0$ such that

$$
\begin{aligned}
& \max \left\{C_{1}, C_{2}\right\} \max \left\{\sup _{t>\xi_{m-1}} e^{-\frac{k t}{2}} \int_{0}^{t} e^{-\frac{k s}{2}} \phi_{\max \{R, \widetilde{R}\}}(s) \mathrm{d} s, \int_{0}^{\xi_{m-1}} e^{-\frac{k s}{2}} \phi_{\max \{R, \widetilde{R}\}}(s) \mathrm{d} s\right\} \\
& +\max \left\{C_{1}, C_{2}\right\} \max \left\{\frac{1}{2}, 2\left(1-e^{-\frac{k \xi_{m-1}}{2}}\right)\right\}\left(1+\frac{M}{k}\right) R<R .
\end{aligned}
$$

Then, problem (1) has a solution $u \in X$ such that

$$
\alpha(t) \leq u(t) \leq \beta(t), \quad \forall t \in[0, \infty) .
$$

Proof We will prove the first case, the second being analogous.

Let $\varepsilon>0$ be such that

$$
\begin{gathered}
\max \left\{C_{1}, C_{2}\right\} \max \left\{\sup _{t>\xi_{m-1}} e^{-\frac{k t}{2}} \int_{0}^{t} e^{-\frac{k s}{2}} \varphi_{\max \{R, \widetilde{R}\}}(s) \mathrm{d} s, \int_{0}^{\xi \xi m-1} e^{-\frac{k s}{2}} \varphi_{\max \{R, \widetilde{R}\}}(s) \mathrm{d} s\right\} \\
+\max \left\{C_{1}, C_{2}\right\} \max \left\{\frac{1}{2}, 2\left(1-e^{-\frac{k \xi_{m-1}}{2}}\right)\right\}\left(\left(1+\frac{M}{k}\right) R+\frac{\varepsilon}{k}(R+\widetilde{R})\right)<R .
\end{gathered}
$$

Consider the modified problem

$$
\left\{\begin{aligned}
u^{\prime \prime}(t)+k u^{\prime}(t)+M u(t)= & f\left(t, \delta(t, u(t)), \frac{\mathrm{d}}{\mathrm{d} t} \delta(t, u(t))\right) \\
& +k u^{\prime}(t)+M u(t)+\varepsilon(u(t)-\delta(t, u(t))), \quad t \in[0, \infty), \\
u(0)=0, \quad u^{\prime}(\infty)= & \sum_{i=1}^{m-1} \alpha_{i} u^{\prime}\left(\xi_{i}\right),
\end{aligned}\right.
$$

where the function $\delta:[0, \infty) \times \mathbb{R} \rightarrow \mathbb{R}$ is given by

$$
\delta(t, u(t))= \begin{cases}\beta(t), & u(t)>\beta(t) \\ u(t), & \alpha(t) \leq u(t) \leq \beta(t) \\ \alpha(t), & u(t)<\alpha(t)\end{cases}
$$

Define now operator $T^{*}: X \rightarrow X$ by

$$
\begin{aligned}
T^{*} u(t)= & \int_{0}^{\infty} G(t, s)\left(f\left(s, \delta(s, u(s)), \frac{\mathrm{d}}{\mathrm{d} s} \delta(s, u(s))\right)\right. \\
& \left.+k u^{\prime}(s)+M u(s)+\varepsilon(u(s)-\delta(s, u(s)))\right) \mathrm{d} s .
\end{aligned}
$$


From Lemma 7, we know that $\frac{\mathrm{d}}{\mathrm{d} s} \delta(s, u(s))$ exists for a.e. $s \in[0, \infty)$. Thus, Lemma 7, together with the Carathéodory condition on the nonlinearity, implies that the integral in the latter expression is well-defined.

Following the same steps as in Lemma 5 , it is easy to prove that if $\left(H_{1}\right)$ holds, then $T^{*}$ is well-defined in $X$ and it is a completely continuous operator.

Moreover, by (10), it is clear that

$$
|\delta(t, u(t))| \leq \widetilde{R} \quad \text { and } \quad\left|\frac{\mathrm{d}}{\mathrm{d} t} \delta(t, u(t))\right| \leq \max \{\|u\|, \widetilde{R}\} \quad \text { for all } t \in[0, \infty) .
$$

Thus, if we consider

$$
D=\{u \in X:\|u\|<R\}
$$

and $u \in D$ then, following analogous steps as in the proof of Theorem 6, it can be deduced that

$$
\begin{aligned}
\left\|T^{*} u\right\| \leq & \max \left\{C_{1}, C_{2}\right\} \max \left\{\sup _{t>\xi_{m-1}} e^{-\frac{k t}{2}} \int_{0}^{t} e^{-\frac{k s}{2}} \varphi_{\max \{R, \widetilde{R}\}}(s) \mathrm{d} s, \int_{0}^{\xi_{m-1}} e^{-\frac{k s}{2}} \varphi_{\max \{R, \widetilde{R}\}}(s) \mathrm{d} s\right\} \\
& +\max \left\{C_{1}, C_{2}\right\} \max \left\{\frac{1}{2}, 2\left(1-e^{-\frac{k \xi_{m-1}}{2}}\right)\right\}\left(\left(1+\frac{M}{k}\right) R+\frac{\varepsilon}{k}(R+\widetilde{R})\right)<R,
\end{aligned}
$$

that is, $T^{*} u \in D$.

Therefore, $T D \subset D$ and, from Theorem $3, T^{*}$ has at least one fixed point in $\mathrm{D}$, which corresponds to a solution of problem (11).

Finally, we will prove that this solution $u$ of problem (11) satisfies

$$
\alpha(t) \leq u(t) \leq \beta(t), \quad \forall t \in[0, \infty)
$$

which implies that it is also a solution of problem (5).

Define $v(t)=u(t)-\beta(t)$ and consider $t_{0} \in[0, \infty) \cup\{\infty\}$ such that

$$
v\left(t_{0}\right):=\sup \{v(t): t \in[0, \infty)\}
$$

Suppose that $v\left(t_{0}\right)>0$. Then, since

$$
\nu(0)=-\beta(0) \leq 0,
$$

necessarily $t_{0} \neq 0$. Thus, there exists $\bar{t} \in[0, \infty)$ such that

$$
v(t)>0 \quad \text { for } t \in\left(\bar{t}, t_{0}\right) \quad \text { and } \quad v^{\prime}(\bar{t}) \geq 0 .
$$

Now, using the facts that $u$ is a solution of (11) and $\beta$ is an upper solution of problem (1), we obtain for $t \in\left(\bar{t}, t_{0}\right)$ that

$$
\begin{aligned}
u^{\prime \prime}(t) & =f\left(t, \delta(t, u(t)), \frac{\mathrm{d}}{\mathrm{d} t} \delta(t, u(t))\right)+\varepsilon(u(t)-\delta(t, u(t))) \\
& =f\left(t, \beta(t), \beta^{\prime}(t)\right)+\varepsilon(u(t)-\beta(t)) \geq \beta^{\prime \prime}(t)+\varepsilon(u(t)-\beta(t)) .
\end{aligned}
$$


Thus, we deduce that

$$
v^{\prime \prime}(t)=u^{\prime \prime}(t)-\beta^{\prime \prime}(t) \geq \varepsilon(u(t)-\beta(t))=\varepsilon v(t)>0, \quad t \in\left(\bar{t}, t_{0}\right)
$$

which implies that $v^{\prime}$ is strictly increasing on $\left(\bar{t}, t_{0}\right)$. In particular, since $v^{\prime}(\bar{t}) \geq 0$, it must be true that $v^{\prime}>0$ on $\left(\bar{t}, t_{0}\right)$.

Now, the fact that $v^{\prime}>0$ on $\left(\bar{t}, t_{0}\right)$ implies that $t_{0}=\infty$ and so

$$
\lim _{t \rightarrow \infty} v^{\prime}(t)>0
$$

On the other hand, since $v \in X$,

$$
v(\infty)=\sup _{t \in[0, \infty)} v(t)=C \in \mathbb{R}
$$

and, using L'Hôpital's Rule,

$$
C=\lim _{t \rightarrow \infty} v(t)=\lim _{t \rightarrow \infty} \frac{e^{t} v(t)}{e^{t}}=\lim _{t \rightarrow \infty} \frac{e^{t} v(t)+e^{t} v^{\prime}(t)}{e^{t}}=\lim _{t \rightarrow \infty} v(t)+v^{\prime}(t)=C+\lim _{t \rightarrow \infty} v^{\prime}(t),
$$

hence we deduce that

$$
\lim _{t \rightarrow \infty} v^{\prime}(t)=0
$$

which is a contradiction.

Therefore

$$
\sup \{v(t): t \in[0, \infty)\} \leq 0
$$

that is,

$$
u(t) \leq \beta(t), \quad t \in[0, \infty) .
$$

Analogously, it can be seen that

$$
u(t) \geq \alpha(t), \quad t \in[0, \infty) .
$$

This way, we conclude that $u$ is a solution of problem (1).

\section{Example}

Let us consider the following boundary value problem:

$$
\left\{\begin{array}{l}
u^{\prime \prime}(t)=\frac{1}{1000}(2+\sin t) e^{-|u(t)|} \frac{|1-u(t)|}{(u(t))^{2}+1}\left(u^{\prime}(t)-1\right), \quad t \in[0, \infty) \\
u(0)=0, \quad u^{\prime}(\infty)=0.11 u^{\prime}(0)+0.89 u^{\prime}(0.11)
\end{array}\right.
$$

This problem is a particular case of $(1)$ with $f(t, x, y)=\frac{1}{1000}(2+\sin t) e^{-|x|} \frac{|1-x|}{x^{2}+1}(y-1), m=3$, $\alpha_{1}=0.11, \alpha_{2}=0.89, \xi_{1}=0$ and $\xi_{2}=0.11$. 
For $|x|,|y|<r$, it holds that

$$
|f(t, x, y)| \leq \frac{1}{1000}(2+\sin t)(r+1)^{2},
$$

so we could take $\phi_{r}(t)=\frac{1}{1000}(2+\sin t)(r+1)^{2}$ and hence hypothesis $\left(H_{2}\right)$ holds. We note that, since $\phi_{r} \notin L^{1}[0, \infty)$, results in [12] cannot be applied to solve this problem.

We will look for a pair of lower and upper solutions of problem (1) and suitable values for $k$ and $M$ for which the hypotheses in Theorem 8 hold.

As lower and upper solutions we will take

$$
\alpha(t)=\frac{3}{400}\left(-(t+1) e^{-t}+\frac{t^{2}-t}{t^{2}+1}\right) \quad \text { and } \quad \beta(t)=1, \quad \forall t \in[0, \infty)
$$

It can be checked that $\|\alpha\|_{\infty} \approx 0.0087,\left\|\alpha^{\prime}\right\|_{\infty} \approx 0.0065$ and $\|\beta\|_{\infty}=1,\left\|\beta^{\prime}\right\|_{\infty}=0$. Therefore, we obtain that $\widetilde{R}$ given in (10) is

$$
\widetilde{R}=1 \text {. }
$$

Moreover, for $M=0.35$ and $k=0.86$, we obtain the following approximations for $C_{1}$ and $C_{2}$ :

$$
C_{1} \approx 1.2305, \quad C_{2} \approx 1.3395
$$

Therefore,

$$
\max \left\{C_{1}, C_{2}\right\} \max \left\{\frac{1}{2}, 2\left(1-e^{-\frac{k \xi m-1}{2}}\right)\right\}\left(1+\frac{M}{k}\right) \approx 0.9423 .
$$

On the other hand,

$$
\int_{0}^{\xi_{m-1}} e^{-\frac{k s}{2}} \phi_{\max \{R, \widetilde{R}\}}(s) \mathrm{d} s \approx 0.00022(\max \{R, \widetilde{R}\}+1)^{2}
$$

and

$$
\sup _{t>\xi_{m-1}} e^{-\frac{k t}{2}} \int_{0}^{t} e^{-\frac{k s}{2}} \phi_{\max \{R, \widetilde{R}\}}(s) \mathrm{d} s \approx 0.00174(\max \{R, \widetilde{R}\}+1)^{2} .
$$

Therefore, we can approximate

$$
\begin{aligned}
\max & \left\{C_{1}, C_{2}\right\} \max \left\{\sup _{t>\xi_{m-1}} e^{-\frac{k t}{2}} \int_{0}^{t} e^{-\frac{k s}{2}} \phi_{\max \{R, \widetilde{R}\}}(s) \mathrm{d} s, \int_{0}^{\xi_{m-1}} e^{-\frac{k s}{2}} \phi_{\max \{R, \widetilde{R}\}}(s) \mathrm{d} s\right\} \\
& +\max \left\{C_{1}, C_{2}\right\} \max \left\{\frac{1}{2}, 2\left(1-e^{-\frac{k \xi_{m-1}}{2}}\right)\right\}\left(1+\frac{M}{k}\right) R \\
\approx & 0.00233(\max \{R, 1\}+1)^{2}+0.9423 R,
\end{aligned}
$$

and it can be seen that for $R \in\left(R_{0}, R_{1}\right)$, with $R_{0} \approx 0.1615$ and $R_{1} \approx 22.7199$, it holds that

$$
0.00233(\max \{R, 1\}+1)^{2}+0.9423 R<R .
$$


Therefore, we have proved the existence of a solution $u$ of problem (12) such that

$$
\frac{3}{400}\left(-(t+1) e^{-t}+\frac{t^{2}-t}{t^{2}+1}\right) \leq u(t) \leq 1, \quad \forall t \in[0, \infty)
$$

which implies that this solution is nontrivial.

\section{Acknowledgements}

Not applicable.

Funding

First author was partially supported by Xunta de Galicia (Spain), project EM2014/032, AEI Spain and FEDER, grants MTM2013-43014-P, MTM2016-75140-P, and FPU scholarship, Ministerio de Educación, Cultura y Deporte, Spain.

Availability of data and materials

Not applicable.

\section{Competing interests}

The authors declare that there is no conflict of interest regarding the publication of this article.

\section{Authors' contributions}

Each author equally contributed to this paper, and read and approved the final manuscript.

\section{Author details}

'Departamento de Estatística, Análise Matemática e Optimización Instituto de Matemáticas, Facultade de Matemáticas, Universidade de Santiago de Compostela, Galicia, Spain. ²Departamento de Matemática, Escola de Ciências e Tecnologia, Centro de Investigação em Matemática e Aplicações (CIMA), Instituto de Investigação e Formação Avançada, Universidade de Évora, Évora, Portugal.

\section{Publisher's Note}

Springer Nature remains neutral with regard to jurisdictional claims in published maps and institutional affiliations.

Received: 21 October 2018 Accepted: 7 February 2019 Published online: 15 February 2019

\section{References}

1. Agarwal, R.P., O'Regan, D.: Infinite Interval Problems for Differential, Difference and Integral Equations (2001)

2. Bai, C., Fang, J.: Existence of positive solutions for three-point boundary value problems at resonance. J. Math. Anal. Appl. 291, 538-549 (2004)

3. Bebernes, J., Gaines, R., Schmitt, K.: Existence of periodic solutions for third and fourth order ordinary differential equations via coincidence degree. Ann. Soc. Sci. Brux. Sér. I 88, 25-36 (1974)

4. Cabada, A.: Green's Functions in the Theory of Ordinary Differential Equations. Springer Briefs in Math. (2014)

5. Corduneanu, C.: Integral Equations and Stability of Feedback Systems. Academic Press, New York (1973)

6. Djebali, S., Guedda, L.: A third order boundary value problem with nonlinear growth at resonance on the half-axis. Math. Methods Appl. Sci. 40(8), 2859-2871 (2017)

7. Feng, W., Webb, J.R.L.: Solvability of three-point boundary value problems at resonance. Nonlinear Anal. 30 3227-3238 (1997)

8. Franco, D., Infante, G., Zima, M.: Second order nonlocal boundary value problems at resonance. Math. Nachr. 284, 875-884 (2011)

9. Han, X.: Positive solutions for a three-point boundary value problem at resonance. J. Math. Anal. Appl. 336, 556-568 (2007)

10. Infante, G., Pietramala, P., Tojo, F.A.F.: Nontrivial solutions of local and nonlocal Neumann boundary value problems. Proc. R. Soc. Edinb. A 146, 337-369 (2016)

11. Jiang, W.: Solvability for p-Laplacian boundary value problem at resonance on the half-line. Bound. Value Probl. 2013, 207 (2013)

12. Jiang, W., Yang, C.: The existence of positive solutions for multi-point boundary value problem at resonance on the half-line. Bound. Value Probl. 2016, 13, 12 (2016)

13. Kaufmann, E.R.: Existence of solutions for a nonlinear second-order equation with periodic boundary conditions at resonance. Commun. Appl. Anal. 18, 163-174 (2014)

14. Kim, C.-G.: Existence and iteration of positive solutions for multi-point boundary value problems on a half-line. Comput. Math. Appl. 61(7), 1898-1905 (2011)

15. Kosmatov, N.: Multi-point boundary value problems on an unbounded domain at resonance. Nonlinear Anal. 68 , 2158-2171 (2008)

16. Lian, H., Ge, W.: Solvability for second-order three-point boundary value problems on a half-line. Appl. Math. Lett. 19(10), 1000-1006 (2006)

17. Ma, R., Yang, Y.: Existence result for a singular nonlinear boundary value problem at resonance. Nonlinear Anal. 68 671-680 (2008)

18. Minhós, F., Carrasco, H.: Existence of homoclinic solutions for nonlinear second-order problems. Mediterr. J. Math. 13 3849 (2016) 
19. Minhós, F., Carrasco, H.: Homoclinic solutions for nonlinear general fourth-order differential equations. Math. Methods Appl. Sci. 40, 5768-5776 (2017)

20. Mosa, S., Eloe, P.: Upper and lower solution method for boundary value problems at resonance. Electron. J. Qual. Theory Differ. Equ. 2016, 40, 1-13 (2016)

21. O'Regan, D., Zima, M.: Leggett-Williams norm-type theorems for coincidences. Arch. Math. 87, 233-244 (2006)

22. Rachunková, I., Staněk, S.: Topological degree method in functional boundary value problems at resonance. Nonlinear Anal. 27, 271-285 (1996)

23. Wang, M.X., Cabada, A., Nieto, J.: Monotone method for nonlinear second order periodic boundary value problems with Carathéodory functions. Ann. Pol. Math. 58, 221-235 (1993)

24. $\mathrm{Xu}, \mathrm{J}$., Yang, Z:: Positive solutions for singular Sturm-Liouville boundary value problems on the half line. Electron. J. Differ. Equ. 2010, 171, 1-8 (2010)

25. Yang, A., Henderson, J., Wang, D.: The study of higher-order resonant and nonresonant boundary value problems. Electron. J. Qual. Theory Differ. Equ. 3, 1 (2016)

26. Zeidler, E.: Nonlinear Functional Analysis and Its Applications. I: Fixed-Point Theorems. Springer, New York (1986) Translated from the German by Peter R. Wadsack

Submit your manuscript to a SpringerOpen ${ }^{\circ}$ journal and benefit from:

- Convenient online submission

- Rigorous peer review

Open access: articles freely available online

- High visibility within the field

- Retaining the copyright to your article

Submit your next manuscript at $\boldsymbol{\nabla}$ springeropen.com 